\title{
Switching in One-Factorisations of Complete Graphs*
}

\author{
Petteri Kaski ${ }^{\dagger}$ \\ Helsinki Institute for Information Technology HIIT \\ Department of Information and Computer Science \\ Aalto University, P.O. Box 15400, 00076 Aalto, Finland \\ petteri.kaski@aalto.fi \\ André de Souza Medeiros \\ Department of Information and Computer Science \\ Aalto University, P.O. Box 15400, 00076 Aalto, Finland \\ andre.medeiros@aalto.fi \\ Patric R. J. Östergård \\ Department of Communications and Networking \\ Aalto University School of Electrical Engineering \\ P.O. Box 13000, 00076 Aalto, Finland \\ patric.ostergard@aalto.fi \\ Ian M. Wanless ${ }^{\S}$ \\ School of Mathematical Sciences \\ Monash University \\ Vic 3800, Australia \\ ian.wanless@monash.edu
}

Submitted: Jul 27, 2013; Accepted: May 27, 2014; Published: Jun 9, 2014

\begin{abstract}
We define two types of switchings between one-factorisations of complete graphs, called factor-switching and vertex-switching. For each switching operation and for each $n \leqslant 12$, we build a switching graph that records the transformations between isomorphism classes of one-factorisations of $K_{n}$. We establish various parameters of our switching graphs, including order, size, degree sequence, clique number and the radius of each component.

As well as computing data for $n \leqslant 12$, we demonstrate several properties that hold for one-factorisations of $K_{n}$ for general $n$. We show that such factorisations have a parity which is not changed by factor-switching, and this leads to disconnected switching graphs. We also characterise the isolated vertices that arise from an absence of switchings. For factor-switching the isolated vertices are perfect onefactorisations, while for vertex-switching the isolated vertices are closely related to atomic Latin squares.
\end{abstract}

*We acknowledge use of computer resources funded by project "Science-IT" at Aalto University.

${ }^{\dagger}$ Research supported by the Academy of Finland grants 252083 and 256287.

${ }^{\ddagger}$ Research supported in part by the Academy of Finland grant 132122 .

${ }^{\S}$ Research supported by Australian Research Council grant FT110100065. 


\section{Introduction}

Certain types of combinatorial objects exist in large numbers despite being constrained by definitions that at first sight require substantial regularity. In particular, this is the case for many types of designs and codes, where the observed abundance may be studied and, to a satisfactory extent, explained by means of switching; that is, by identifying local substructures that may be transformed to obtain new objects with the same parameters [17]. As well as giving structural insights, switching can be used for enumeration [7] and random generation [9]. The objective of this paper is to initiate a systematic study of switching in the context of one-factorisations of the complete graph $K_{n}$.

We define two classes of natural switching operations, one based on the one-factors and another that is based on the vertices. We also consider two other classes of switchings which correspond to the intersection and the union of the first two classes. For each class of switching operation we define a switching graph, which is a graph with one vertex for each isomorphism class of one-factorisations of $K_{n}$, and edges indicating that switching can transform a factorisation in one isomorphism class into some factorisation in the other class. We then investigate graph-theoretic parameters of our switching graphs as a means to study the relationships between different one-factorisations and the power of switching to explain the observed abundance of isomorphism classes. The number of isomorphism classes of one-factorisations of $K_{n}$ goes to infinity as the order $n$ goes to infinity [12]. The number of isomorphism classes is known exactly for $n \leqslant 14$ [11]. Since the number of isomorphism classes for $n=14$ is too large for an exhaustive explicit study of the classes, we consider $n \leqslant 12$ in the current study.

To place our paper into context in terms of related work, one-factorisations of $K_{n}$ may be viewed as a family of group divisible designs (GDDs), which are discussed and surveyed from a switching perspective in [17, Sect. 2.5]. A $(k, \lambda)$-GDD of type $g_{1}^{a_{1}} g_{2}^{a_{2}} \cdots g_{p}^{a_{p}}$ is a triple $(X, \mathcal{G}, \mathcal{B})$, where $X$ is a set of $\sum_{i=1}^{p} a_{i} g_{i}$ points, $\mathcal{G}$ is a partition of $X$ into $a_{i}$ subsets of size $g_{i}$ for $1 \leqslant i \leqslant p$ (known as groups), and $\mathcal{B}$ is a collection of $k$-subsets (known as blocks) of points, such that every 2 -subset of points occurs in exactly $\lambda$ blocks or one group, but not both. If $\lambda=1$, we may simply write $k$-GDD. There are three main types of combinatorial objects that can be considered within the framework of 3-GDDs: Latin squares (type $n^{3}$ for order $n$ ), Steiner triple systems (type $1^{v}$ for order $v$ ), and one-factorisations of the complete graph (type $(n-1)^{1} 1^{n}$ for order $n$ ). For the former two types of objects, extensive studies of switching and related properties have been carried out to the computational limit, see [23] and [5, 8, 10], respectively. Studies demonstrating the wider importance of switching of these types of objects include $[2,4]$. However, as pointed out in [10] and [17], a study of switchings for one-factorisations of complete graphs has been missing. The current study fills that gap.

The outline of the paper is as follows. In Section 2 we collect the basic definitions and notation that is used throughout the paper. There are a few more possibilities of switching for one-factorisations of a complete graph than for Latin squares and Steiner triple systems. Possible switching operations are discussed in Section 3. In Section 4 it is shown that for some types of switchings, the switching graph will be disconnected for 
all orders because one-factorisations have a parity that is not changed by the switchings. The computational task of constructing switching graphs is discussed in Section 5. The remainder of the paper presents various properties of the switching graphs including degree sequence and components (Section 6), cliques (Section 7) and the radius and diameter of each component (Section 8).

\section{Basic definitions}

In this section we collect the basic definitions that are used throughout the paper.

A $k$-factor of a graph $G$ is a $k$-regular spanning subgraph of $G$. The edges of a onefactor form a perfect matching, which is a set of edges such that every vertex of $G$ is incident with exactly one edge in the set. A prerequisite for the existence of a one-factor is that $G$ has even order. A one-factorisation of $G$ is a decomposition of $G$ into onefactors. In other words, it is a set of one-factors of $G$ such that every edge of $G$ is in exactly one of the one-factors.

Example 1. A one-factorisation of $K_{8}$.

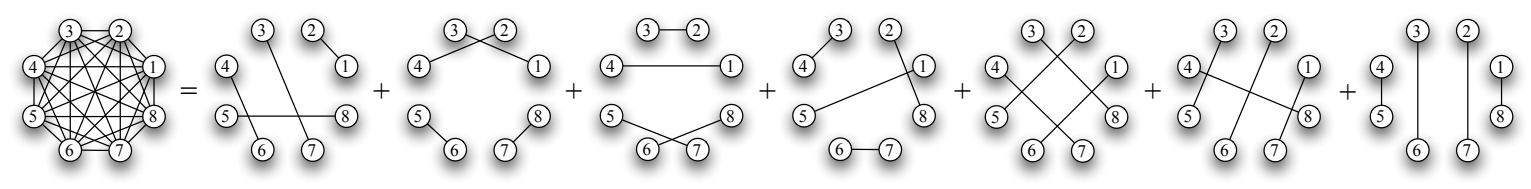

An alternative viewpoint of one-factorisations is that a one-factorisation of a $k$-regular graph $G$ is a proper edge colouring of $G$ with $k$ colours, where each colour induces a one-factor. Since the one-factorisation is an unordered set of one-factors, renaming the colours does not change the one-factorisation. In this paper, unless otherwise specified, the term "one-factorisation" will mean a one-factorisation of the complete graph $K_{n}$ using $\{1,2, \ldots, n-1\}$ as the set of colours.

Suppose $\mathscr{F}$ is a one-factorisation of a graph $G$. The union of any two one-factors from $\mathscr{F}$ gives a two-factor of $G$. Since the two-factor is edge 2-colourable, it is necessarily a union of even-length cycles. We will refer to these cycles as the cycles of the onefactorisation $\mathscr{F}$. If all the cycles of $\mathscr{F}$ are Hamiltonian cycles of $G$ then we say that $\mathscr{F}$ is a perfect one-factorisation $(P 1 F)$.

A Latin square of order $n$ is an $n \times n$ matrix containing $n$ symbols in such a way that every symbol occurs exactly once in each row and exactly once in each column. A Latin subrectangle of a Latin square is a rectangular submatrix in which the same set of symbols occurs in each row. A $2 \times 2$ Latin subrectangle (subsquare) is known as an intercalate.

A Latin square is symmetric if it equals its matrix transpose, and it is unipotent if only one symbol occurs on the main diagonal. There is a natural way to write a onefactorisation $\mathscr{F}$ of $K_{n}$ as a symmetric unipotent Latin square of order $n$, which we will denote $U_{\mathscr{F}}$. Label the vertices of the complete graph $v_{1}, v_{2}, \ldots, v_{n}$. Now, define the offdiagonal entries of $U_{\mathscr{F}}$ by $U_{\mathscr{F}}[i, j]=k$ if the edge $v_{i} v_{j}$ has colour $k$ in $\mathscr{F}$. The diagonal 
entries in $U_{\mathscr{F}}$ will always be denoted by $\cdot$ in this paper, but this can be interpreted as being the symbol $n$.

We have just explained how each one-factorisation $\mathscr{F}$ of $K_{n}$ corresponds to a unipotent Latin square $U_{\mathscr{F}}$ of order $n$. Each such Latin square corresponds to $n$ potentially different Latin squares of order $n-1$. Select any $x$ in the range $1 \leqslant x \leqslant n$ and copy $U_{\mathscr{F}}[x, i]$ to $U_{\mathscr{F}}[i, i]$ for $1 \leqslant i \leqslant n$, then delete row $x$ and column $x$ to obtain a matrix we denote by $U_{\mathscr{F}, x}$. Since $U_{\mathscr{F}}$ is a symmetric unipotent Latin square of order $n$, it follows that $U_{\mathscr{F}, x}$ is a symmetric Latin square of order $n-1$. In general, the $n$ possible choices of $x$ result in $n$ quite different Latin squares. In the terminology of [3], these $n$ Latin squares are folds of each other. The relationships between $\mathscr{F}, U_{\mathscr{F}}$ and $U_{\mathscr{F}, x}$ are studied in detail in [25].

Consider a one-factorisation $\mathscr{F}$ of $K_{n}$. An isomorphic one-factorisation is one obtained by permuting the labels on the vertices of $K_{n}$. The automorphism group of $\mathscr{F}$ is the set of such permutations that produce the same one-factorisation (up to relabelling of the colours). There is an isomorphism between $\mathscr{F}$ and $\mathscr{F}^{\prime}$ if and only if $U_{\mathscr{F}}$ can be obtained by applying one permutation simultaneously to the rows and columns of $U_{\mathscr{F} \prime}$, then applying a second permutation to the symbols $\{1,2, \ldots, n-1\}$.

Suppose that $L=\left[L_{i j}\right]$ is a Latin square of order $n$. Associated with $L$, we have a set of $n^{2}$ triples $\left(i, j, L_{i j}\right)$ for $1 \leqslant i, j \leqslant n$. Conjugates of $L$ are formed by uniformly permuting these triples, then interpreting the result as a new Latin square. There are 6 conjugates, corresponding to the 6 permutations in $S_{3}$. Corresponding to $L$ we also have $n$ row permutations $\sigma_{i}$ defined by $\sigma_{i}(j)=L_{i j}$. Each $\sigma_{i}$ has a parity in the usual sense for permutations. If $L$ has an odd number of rows for which $\sigma_{i}$ is an odd permutation then we say it is row-odd, otherwise it is row-even. Column-odd and column-even are defined similarly. For more background on the parity of Latin squares, see [20, 23].

Suppose $1 \leqslant i<i^{\prime} \leqslant n$ and consider the cycles of the permutation $\sigma_{i} \sigma_{i^{\prime}}^{-1}$. Such a cycle, say $\left(x_{1} x_{2} \cdots x_{\ell}\right)$, corresponds to a $2 \times \ell$ Latin subrectangle $R$ inside $L$ that contains all occurences of the symbols $x_{1}, x_{2}, \ldots, x_{\ell}$ in rows $i$ and $i^{\prime}$. Moreover, $R$ is minimal in the sense that it does not contain any $2 \times \ell^{\prime}$ Latin subrectangle for $0<\ell^{\prime}<\ell$. We call $R$ a row cycle of length $\ell$ in $L$. It is possible to switch the rows of $R$ to obtain a new Latin square from $L$. This process is called row cycle switching. There are corresponding notions of column cycle switching and symbol cycle switching, which are related to row cycle switching by the conjugacy operation on Latin squares. For full details see [23].

$$
\left[\begin{array}{llllllll}
\cdot & 1 & 2 & 3 & 4 & 5 & 6 & 7 \\
1 & . & 3 & 2 & 5 & 6 & 7 & 4 \\
2 & 3 & . & 4 & 6 & 7 & 1 & 5 \\
3 & 2 & 4 & . & 7 & 1 & 5 & 6 \\
4 & 5 & 6 & 7 & . & 2 & 3 & 1 \\
5 & 6 & 7 & 1 & 2 & . & 4 & 3 \\
6 & 7 & 1 & 5 & 3 & 4 & . & 2 \\
7 & 4 & 5 & 6 & 1 & 3 & 2 & \cdot
\end{array}\right]
$$

(a)

$\left[\begin{array}{llllllll}\cdot & 1 & 2 & 3 & 4 & 5 & 6 & 7 \\ 1 & \cdot & 5 & 2 & 3 & 6 & 7 & 4 \\ 2 & 5 & \cdot & 4 & 6 & 7 & 3 & 1 \\ 3 & 2 & 4 & \cdot & 7 & 1 & 5 & 6 \\ 4 & 3 & 6 & 7 & . & 2 & 1 & 5 \\ 5 & 6 & 7 & 1 & 2 & \cdot & 4 & 3 \\ 6 & 7 & 3 & 5 & 1 & 4 & \cdot & 2 \\ 7 & 4 & 1 & 6 & 5 & 3 & 2 & \cdot\end{array}\right]$

(b) 
Example 2. In (a) above we show $U_{\mathscr{F}}$ where $\mathscr{F}$ is the one-factorisation given in Example 1. To obtain (b) we have switched the darkly shaded row cycle of length 3, and the (symmetrically positioned) lightly shaded column cycle.

We say that a Latin square $L$ of order $n$ is row-Hamiltonian if all of its row cycles have length $n$. Column-Hamiltonian and symbol-Hamiltonian are defined similarly in terms of the lengths of the column cycles and symbol cycles respectively. Row, column or symbol-Hamiltonian Latin squares are necessarily of odd order [22]. We say that $L$ is atomic if all six conjugates of $L$ are row-Hamiltonian; in other words, $L$ is row, column and symbol-Hamiltonian. Row-hamiltonian and atomic Latin squares are closely related to perfect one-factorisations of complete graphs and complete bipartite graphs. For more details, see $[3,13,24]$.

\section{The switching operations}

We next define the switching operations that allow us to convert a one-factorisation $\mathscr{F}$ of $K_{n}$ into a different one-factorisation $\mathscr{F}^{\prime}$ of the same graph. These operations will be used to produce the edges in our switching graphs.

The first switching operation we define is called factor-switching. We choose any two distinct one-factors $f_{1}, f_{2} \in \mathscr{F}$. The union of $f_{1}$ and $f_{2}$ is a bipartite two-factor, and hence consists of vertex-disjoint cycles of even length. Suppose we choose one of these cycles $C$. Within $C$ we can put all the edges that were in $f_{1}$ into $f_{2}$ and vice versa, and this gives us $\mathscr{F}^{\prime}$. If $f_{1} \cup f_{2}$ is a Hamilton cycle then we do not allow factor-switching because the result would obviously be isomorphic. Hence there is some choice of $f_{1}, f_{2}$ for which factor-switchings are available if and only if $\mathscr{F}$ is not a P1F.

The second switching operation is called vertex-switching. We choose two distinct vertices $v_{1}$ and $v_{2}$ of our complete graph $K_{n}$. Suppose the edge $v_{1} v_{2}$ is in factor $f$ in $\mathscr{F}$. We then form a bipartite graph $G$ whose vertices in one part are the vertices of $K_{n}$ other than $v_{1}, v_{2}$, and in the other part are the factors in $\mathscr{F} \backslash\{f\}$. We put an edge in $G$ between $v_{i}$ and $f_{j}$ if $f_{j}$ contains either of the edges $v_{1} v_{i}$ or $v_{2} v_{i}$ of $K_{n}$. By construction, $G$ is a 2-regular bipartite graph, so it consists of disjoint cycles of even length. We can choose any one of these cycles, say $C$, and switch on it. Thus, if $v_{i}$ is connected to $f_{j}$ in $C$ then we replace $v_{1} v_{i}$ by $v_{2} v_{i}$ or vice versa in $f_{j}$. This gives us our new one-factorisation $\mathscr{F}^{\prime}$. Again, we do not allow the switching to occur if $G$ consists of a single cycle, since in that case it would be obvious that $\mathscr{F}^{\prime}$ is isomorphic to $\mathscr{F}$.

The switching operations can be considered in the framework of [17], viewing onefactorisations of $K_{n}$ as GDDs of type $(n-1)^{n} 1^{n}$. Switching in [17] is considered via the particularisation of two points. We get factor-switching if the two points are amongst the $n-1$ points of the GDD corresponding to the one-factors, and vertex-switching if they are amongst the $n$ points corresponding to vertices.

Our switching operations on a one-factorisation $\mathscr{F}$ can also be described quite naturally in terms of switching operations on $U_{\mathscr{F}}$ using the terminology from [23] mentioned in Section 2. Factor-switchings are achieved by switching two symbol cycles, whereas vertex- 
switchings are achieved by switching a row cycle and a column cycle. In both cases the two cycles that are switched are symmetrically placed with respect to the main diagonal, and do not intersect that diagonal. In vertex-switching the two rows in the row cycle and columns in the column cycle correspond to the two vertices chosen for the switching. In factor-switching the symbol cycle involves the two symbols (colours) that label the factors chosen for the switching.

Example 3. Below we give the unipotent Latin squares for two one-factorisations of $K_{10}$. In (a) the entries involved in a vertex switching on a cycle of length 6 are shown. They consist of a row cycle of length 3 (shaded lightly), and the corresponding column cycle (shaded darkly). Meanwhile (b) shows the entries for a factor switching on a cycle of length 6 . They consist of two symmetrically placed symbol cycles of length 3.

$$
\left[\begin{array}{llllllllll}
\cdot & 1 & 2 & 3 & 4 & 5 & 6 & 9 & 8 & 7 \\
1 & \cdot & 3 & 2 & 5 & 4 & 9 & 6 & 7 & 8 \\
2 & 3 & \cdot & 1 & 6 & 8 & 4 & 7 & 5 & 9 \\
3 & 2 & 1 & . & 7 & 9 & 8 & 5 & 6 & 4 \\
4 & 5 & 6 & 7 & . & 1 & 2 & 8 & 9 & 3 \\
5 & 4 & 8 & 9 & 1 & . & 7 & 3 & 2 & 6 \\
6 & 9 & 4 & 8 & 2 & 7 & . & 1 & 3 & 5 \\
9 & 6 & 7 & 5 & 8 & 3 & 1 & . & 4 & 2 \\
8 & 7 & 5 & 6 & 9 & 2 & 3 & 4 & \cdot & 1 \\
7 & 8 & 9 & 4 & 3 & 6 & 5 & 2 & 1 & \cdot
\end{array}\right] \quad\left[\begin{array}{llllllllll}
\cdot & 1 & 2 & 4 & 3 & 7 & 5 & 9 & 6 & 8 \\
1 & . & 3 & 2 & 4 & 8 & 9 & 6 & 5 & 7 \\
2 & 3 & . & 1 & 5 & 9 & 7 & 4 & 8 & 6 \\
4 & 2 & 1 & . & 6 & 3 & 8 & 7 & 9 & 5 \\
3 & 4 & 5 & 6 & \cdot & 1 & 2 & 8 & 7 & 9 \\
7 & 8 & 9 & 3 & 1 & \cdot & 6 & 5 & 2 & 4 \\
5 & 9 & 7 & 8 & 2 & 6 & . & 1 & 4 & 3 \\
9 & 6 & 4 & 7 & 8 & 5 & 1 & \cdot & 3 & 2 \\
6 & 5 & 8 & 9 & 7 & 2 & 4 & 3 & \cdot & 1 \\
8 & 7 & 6 & 5 & 9 & 4 & 3 & 2 & 1 & \cdot
\end{array}\right]
$$

$(a)$

It is obvious from the effect on $U_{\mathscr{F}}$ that there are some switchings that are both factorswitchings and vertex-switchings. These are the examples where the switching takes place on a cycle $C$ of length 4 . (The length we quote here is the length of the cycle in the graph used to construct the switching. A graphical 4-cycle corresponds to a row cycle of length 2 , which is the same thing as a column cycle of length 2 or a symbol cycle of length 2.) These 4-cycle switchings correspond naturally to the intercalate switchings in [23] and also to Pasch switches [17]. In recognition of their importance, our first switching graph allows only 4-cycle switchings.

We shall now define four types of switching graphs with vertices that are the isomorphism classes of one-factorisations of a complete graph $K_{n}$. The graphs differ only in the switching operations that are allowed to produce edges. We do not allow multiple edges. We do allow loops, but ignore them for the purpose of counting degrees. The graphs are:

$\mathscr{G}_{n}^{\mathrm{i}}$ Edges are produced by switchings that are simultaneously factor-switchings and vertex-switchings (in other words, they are 4-cycle switchings).

$\mathscr{G}_{n}^{\mathrm{f}}$ Edges are produced by factor-switchings.

$\mathscr{G}_{n}^{\mathrm{v}}$ Edges are produced by vertex-switchings.

$\mathscr{G}_{n}^{\mathrm{fv}}$ Edges are produced by factor-switchings and also by vertex-switchings. In other words the edge set of $\mathscr{G}_{n}^{\mathrm{fv}}$ is the union of the edge sets for $\mathscr{G}_{n}^{\mathrm{v}}$ and $\mathscr{G}_{n}^{\mathrm{f}}$. 
For very small orders, some of these graphs coincide. For $n \leqslant 6$ the only vertexswitchings are 4-cycle switchings. Also, for $n \leqslant 10$ all factor-switchings produce a result isomorphic to a 4-cycle switching. To see this, note that if the union of two one-factors forms just two cycles, then switching on either cycle produces the same result (up to isomorphism). So to have a factor-switching available that is not equivalent to a 4-cycle switching, there must be at least 12 vertices. Any 4-cycle switching can be interpreted as either a factor-switching or vertex-switching. The above observations mean that $\mathscr{G}_{n}^{\mathrm{i}}=\mathscr{G}_{n}^{\mathrm{f}}$ and $\mathscr{G}_{n}^{\mathrm{v}}=\mathscr{G}_{n}^{\mathrm{fv}}$ for $n \leqslant 10$, and that all four graphs coincide for $n \leqslant 6$.

Throughout this paper, we will abuse terminology slightly by saying that a particular one-factorisation or its corresponding unipotent Latin square is a vertex of a switching graph, when in fact it is the isomorphism class of the one-factorisation that is the vertex.

\section{Parity and perfection}

Each one-factorisation $\mathscr{F}$ has a parity that we can determine by looking at the corresponding unipotent Latin square $U_{\mathscr{F}}$. If $U_{\mathscr{F}}$ is row-even then we say that $\mathscr{F}$ is even, otherwise we say that $\mathscr{F}$ is $o d d$. Note that $U_{\mathscr{F}}$ is necessarily symmetric, so it is row-even if and only if it is column-even. For this reason, we only need to consider two possible parities for one-factorisations. In contrast, for Latin squares there are eight possible parities, only four of which can be achieved for any particular order [20, 23].

For $n \leqslant 6$ our switching graphs consist of a single vertex. We next show that, except for these trivial cases, there are two types of switching graphs that are always disconnected.

Theorem 1. The graphs $\mathscr{G}_{n}^{\mathrm{i}}$ and $\mathscr{G}_{n}^{\mathrm{f}}$ are disconnected for all even $n \geqslant 8$.

Proof. Since the edges of $\mathscr{G}_{n}^{\mathrm{i}}$ are a subset of those for $\mathscr{G}_{n}^{\mathrm{f}}$, it suffices to prove the result for $\mathscr{G}_{n}^{\mathrm{f}}$. Suppose $m, n$ are positive even integers. It is well known that a one-factorisation of $K_{m}$ can be embedded in some one-factorisation of $K_{n}$ if and only if $n \geqslant 2 m$ (for a proof, see [12] or [21, Thm 14.2]). Thus for $n \geqslant 16$ there exists a one-factorisation of $K_{n}$ containing the one-factorisation in Example 2(a). Replacing this sub one-factorisation by the one-factorisation in Example 2(b) changes the parity. Hence for $n=8$ and $n \geqslant 16$ there exist one-factorisations of $K_{n}$ of each parity. It is easy to find examples of both parities for $n \in\{10,12,14\}$ as well. When $n=10$ we may take the factorisation given in Example 3(a), and the one obtained from it by the indicated vertex switching. For $n \in\{12,14\}$ the P1Fs contain examples of both parities.

Finally, we argue that factor-switching never changes the parity of a one-factorisation. Suppose we factor-switch from a one-factorisation $\mathscr{F}$ to a one-factorisation $\mathscr{F}^{\prime}$, by switching a cycle $C$ of length $\ell$ formed by the union of one-factors with colours $a$ and $b$. Then to convert $U_{\mathscr{F}}$ into $U_{\mathscr{F} \prime}$, we transpose the symbols $a$ and $b$ in the $\ell$ rows corresponding to the vertices in $C$. Since $C$ is bipartite, $\ell$ is even, so $U_{\mathscr{F}}$ and $U_{\mathscr{F}}$ have the same row-parity. So there is no way for a factor-switching to convert an even one-factorisation into an odd one-factorisation or vice versa. We conclude that $\mathscr{G}_{n}^{\mathrm{f}}$ is disconnected for $n \geqslant 8$. 
There is no corresponding result for the graphs $\mathscr{G}_{n}^{\mathrm{v}}$ or $\mathscr{G}_{n}^{\mathrm{fv}}$. We can see from Examples 2 and 3 that vertex switchings can change the parity of a one-factorisation. Indeed, they will change the parity if and only if the row-cycle and column-cycle where the switching takes place have odd length.

An result analogous to Theorem 1 was shown for Latin squares in [23], where the overwhelming majority of Latin squares of a given parity were found to be connected to each other by switchings. This led to large components of roughly equal order in the switching graphs. For further discussion on why there are similar number of Latin squares of each parity, see [20]. We will see similar behaviour for our one-factorisations in Section 6.

In addition to the large components observed in the switching graphs in [23], there were often a number of very small components, including isolated vertices. There are two distinct reasons why isolated vertices might occur. One is that there are no switching operations available, which results in a loopless isolated vertex. On the other hand, it is plausible that there could be switching operations available but they all produce a onefactorisation that is isomorphic to the one you started with. This situation would create an isolated vertex with a loop, but in practice it never arose in the one-factorisations studied in this paper. This represents a distinction between the present situation and the switchings studied in [23]. However, the loopless isolated vertices are still of interest, so we next characterise them.

Theorem 2. Suppose $\mathscr{F}$ is a one-factorisation of $K_{n}$ and let $x$ denote a vertex of $K_{n}$. The following statements are equivalent to each other:

1. There are no 4-cycle switchings available from $\mathscr{F}$.

2. $U_{\mathscr{F}}$ has exactly $\left(\begin{array}{l}n \\ 2\end{array}\right)$ intercalates.

3. $U_{\mathscr{F}, x}$ has no intercalates, for all choices of $x$.

Also, the following statements are equivalent to each other:

4. There are no factor-switchings available from $\mathscr{F}$.

5. $\mathscr{F}$ is perfect.

6. $U_{\mathscr{F}, x}$ is symbol-Hamiltonian for some choice of $x$.

7. $U_{\mathscr{F}, x}$ is symbol-Hamiltonian for all choices of $x$.

Moreover, the following statements are equivalent to each other:

8. There are no vertex-switchings available from $\mathscr{F}$.

9. $U_{\mathscr{F}, x}$ is row-Hamiltonian for all choices of $x$.

10. $U_{\mathscr{F}, x}$ is column-Hamiltonian for all choices of $x$. 
Proof. By definition, a P1F $\mathscr{F}$ of $K_{n}$ has the property that the union of any two onefactors in $\mathscr{F}$ produces a Hamiltonian cycle. This is exactly the situation in which there are no factor switchings available from $\mathscr{F}$ (since none could possibly reach a one-factorisation outside the isomorphism class of $\mathscr{F})$. Hence statements 4 and 5 are equivalent. The equivalence of conditions 5, 6 and 7 is shown in [25].

Suppose there is a vertex-switching available from $\mathscr{F}$, based on the vertices $v_{1}, v_{2}$. Then $U_{\mathscr{F}}$ has a row cycle $R$ in the rows corresponding to $v_{1}$ and $v_{2}$, such that $R$ does not intersect the main diagonal of $U_{\mathscr{F}}$. Also, for $R$ to be part of an available switch it must have length strictly less than $n-2$, so there is some column that does not correspond to $v_{1}$ or $v_{2}$, and does not intersect $R$. Let $x$ be the index of this column, and consider $U_{\mathscr{F}, x}$. Since $R$ does not intersect row $x$, column $x$ or the main diagonal of $U_{\mathscr{F}}$, there is an intact copy of $R$ in $U_{\mathscr{F}, x}$. Hence $U_{\mathscr{F}, x}$ is not row-Hamiltonian, which shows that statement 9 implies statement 8. Conversely, suppose $U_{\mathscr{F}, x}$ is not row-Hamiltonian for some particular $x$. Then there are rows $r_{1}, r_{2}$ of $U_{\mathscr{F}, x}$ in which there are at least two separate row cycles. Since $U_{\mathscr{F}, x}$ is symmetric, at most one of these row cycles intersects the main diagonal, so there is a row cycle $R$ that does not intersect the main diagonal. A copy of $R$ will be present in $U_{\mathscr{F}}$, meaning that there is a vertex switching available from $\mathscr{F}$. Hence statement 8 implies statement 9 . The fact that $U_{\mathscr{F}, x}$ is symmetric shows that statements 9 and 10 are equivalent.

Finally, consider 4-cycle switching as a special case of vertex switching, using a row cycle that is an intercalate. From our above discussion it is then clear that statements 1 , 2 and 3 are equivalent, given two additional observations. Firstly, $U_{\mathscr{F}}$ is symmetric and unipotent so its $\left(\begin{array}{l}n \\ 2\end{array}\right)$ principal $2 \times 2$ submatrices must be intercalates. Secondly, in any two rows of $U_{\mathscr{F}, x}$ the unique row cycle that meets the main diagonal cannot be an intercalate, since the symbols that occur on the main diagonal are all distinct.

Corollary 1. A one-factorisation $\mathscr{F}$ of $K_{n}$ is perfect if and only if it is a loopless isolated vertex in $\mathscr{G}_{n}^{\mathrm{i}}$ and $\mathscr{G}_{n}^{\mathrm{f}}$. Also, $U_{\mathscr{F}, x}$ is atomic for all $x$ if and only if $\mathscr{F}$ is a loopless isolated vertex in $\mathscr{G}_{n}^{\mathrm{i}}, \mathscr{G}_{n}^{\mathrm{f}}, \mathscr{G}_{n}^{\mathrm{v}}$ and $\mathscr{G}_{n}^{\mathrm{fv}}$.

It is worth noting that [3] contains constructions for one-factorisations $\mathscr{F}$ of complete graphs for which $U_{\mathscr{F}, x}$ is atomic for some but not all choices of $x$, and other examples where $U_{\mathscr{F}, x}$ is atomic for all choices of $x$. These constructions yield:

Corollary 2. Let $n=p+1$ for a prime $p \geqslant 11$. Then $\mathscr{G}_{n}^{\mathrm{i}}$ and $\mathscr{G}_{n}^{\mathrm{f}}$ each contain at least two loopless isolated vertices, whereas $\mathscr{G}_{n}^{\mathrm{v}}$ and $\mathscr{G}_{n}^{\mathrm{fv}}$ each contain at least one loopless isolated vertex. If 2 is a primitive root modulo $p$, then $\mathscr{G}_{n}^{\mathrm{v}}$ and $\mathscr{G}_{n}^{\mathrm{fv}}$ each contain at least two loopless isolated vertices.

A folklore conjecture, known as the Perfect One-Factorisation Conjecture, is that there exists a P1F of $K_{n}$ for all even $n$. If true, this would imply that $\mathscr{G}_{n}^{\mathrm{i}}$ and $\mathscr{G}_{n}^{\mathrm{f}}$ always have a loopless isolated vertex corresponding to the P1F. Meszka [16] constructs onefactorisations of $K_{n}$ that avoid cycles of any one given length $k<n$. Applying his result when $k=4$ yields:

Corollary 3. For all even $n$ there is at least one loopless isolated vertex in $\mathscr{G}_{n}^{\mathrm{i}}$. 
It remains an open question whether there is any switching graph for one-factorisations of $K_{n}$ for $n \geqslant 14$ in which there is an isolated vertex with a loop.

\section{Constructing the graphs}

The algorithm for constructing our switching graphs closely parallels the algorithm in [10]. To start with, we assume that we have available a listing that contains exactly one one-factorisation $\mathscr{F}$ from every isomorphism class of one-factorisations of $K_{n}$. For this task we can use, for example, the algorithms in [6,11]. Suppose there are $N$ different isomorphism classes. From a computational perspective the hardest case for us is $n=12$, where $N=526915620$.

To construct the switching graph, we must be able to efficiently rank the $N$ isomorphism classes. That is, for a given one-factorisation $\mathscr{F}$ of $K_{n}$, we must be able to efficiently compute a number $R(\mathscr{F})$ in the range $1,2, \ldots, N$ such that any two one-factorisations of $K_{n}$ are isomorphic if and only if they are assigned the same number. We build such an algorithm $R$ in two steps.

First, we put each representative of an isomorphism class into canonical form by encoding the one-factorisation as a graph and then running nauty [14, 15]. We encode a one-factorisation $\mathscr{F}$ as a vertex-coloured graph as follows. We start with $K_{n}$, and colour all vertices blue. We then subdivide each edge by inserting a red vertex in the middle. Next, we insert an edge between each pair of red vertices if and only if the corresponding edges of $K_{n}$ belong to the same one-factor of $\mathscr{F}$. It is readily checked that two such coloured graphs are isomorphic if and only if the originating one-factorisations are isomorphic. To speed up the operation of nauty, we further split the red cliques of size $n / 2$ (that is, the one-factors) into distinct colour classes based on the number of occurrences of each isomorphism type of two-factors that the one-factor forms with the other one-factors of $\mathscr{F}$.

Next, we use randomized construction to obtain an injective hash function that maps the $N$ canonical forms into distinct 64 -bit values. Here it is convenient to represent a onefactorization in canonical form as a 3-GDD consisting of $\left(\begin{array}{l}n \\ 2\end{array}\right)$ triples and $n+(n-1)$ points, each triple indicating the incidence of an edge with a one-factor. In total $\left(\begin{array}{l}n \\ 2\end{array}\right)(n-1)$ distinct triples may occur in the canonical forms; with each such triple we associate a uniform random 64-bit value, and the hash value of a canonical form is the exclusive-or of the values of its triples. Given that $N=526915620$ and $N^{2}$ is much less than $2^{64}$, we expect the $N$ hash values of the canonical forms to be distinct, which was indeed verified by computing and sorting the values into lexicographic order.

Given $\mathscr{F}$ as input, we can now determine $R(\mathscr{F})$ as the lexicographic rank of the 64-bit hash value of the canonical form of $\mathscr{F}$. We refer to [10] for a more detailed discussion.

Once the rank algorithm is available, constructing the switching graph for each type of switching operation becomes straightforward. Suppose that $\mathscr{F}$ has rank $i$. We consider all possible ways to switch $\mathscr{F}$ to obtain $\mathscr{F}^{\prime}$. For each such $\mathscr{F}^{\prime}$, we compute $R\left(\mathscr{F}^{\prime}\right)=j$. If $i \neq j$, we join the vertices $i$ and $j$ in the graph; otherwise we record that there is a loop (self-switch) from the class $i$ to itself. If parallel edges arise, we only keep one of them. 


\begin{tabular}{lrrrr}
\hline & $\mathscr{G}_{8}^{\mathrm{i}}=\mathscr{G}_{8}^{\mathrm{f}}$ & $\mathscr{G}_{8}^{\mathrm{v}}=\mathscr{G}_{8}^{\mathrm{fv}}$ & $\mathscr{G}_{10}^{\mathrm{i}}=\mathscr{G}_{10}^{\mathrm{f}}$ & $\mathscr{G}_{10}^{\mathrm{v}}=\mathscr{G}_{10}^{\mathrm{fv}}$ \\
\hline Vertices & 6 & 6 & 396 & 396 \\
Non-loop edges & 5 & 6 & 1667 & 5212 \\
Components & 2 & 1 & 3 & 1 \\
Isolated vertices & 1 & 0 & 1 & 0 \\
Loops & 2 & 2 & 76 & 120 \\
\hline
\end{tabular}

Table 1: The switching graphs for $n=8$ and $n=10$

\begin{tabular}{lrrrr}
\hline & \multicolumn{1}{c}{$\mathscr{G}_{12}^{\mathrm{i}}$} & \multicolumn{1}{c}{$\mathscr{G}_{12}^{\mathrm{f}}$} & \multicolumn{1}{c}{$\mathscr{G}_{12}^{\mathrm{v}}$} & \multicolumn{1}{c}{$\mathscr{G}_{12}^{\mathrm{fv}}$} \\
\hline Vertices & 526915620 & 526915620 & 526915620 & 526915620 \\
Non-loop edges & 4329000960 & 5869920634 & 17972340034 & 19513133875 \\
Components & 199 & 7 & 9 & 3 \\
Isolated vertices & 185 & 5 & 8 & 2 \\
Loops & 351595 & 546796 & 516801 & 711235 \\
\hline
\end{tabular}

Table 2: The switching graphs for $n=12$

In practice we use the following file format to store the graphs: for each vertex $i$, we record first the number of vertices $j$ adjacent to $i$ such that $i<j$, followed by the list of such vertices $j$. The representation of the graph is the concatenation of such records, one for each $i$, which results in a space requirement of $N+E$ words for the graph, where $E$ is the number of edges in the graph. In practice 32-bit words suffice for our purposes since $N \leqslant 2^{32}$. The four graphs $\mathscr{G}_{12}^{\mathrm{i}}, \mathscr{G}_{12}^{\mathrm{f}}, \mathscr{G}_{12}^{\mathrm{v}}, \mathscr{G}_{12}^{\mathrm{fv}}$ require approximately $18,24,69,75$ gigabytes of space and were generated in slightly less than 2 core-years of computing time on a cluster comprising of 118 nodes with two 2.67-GHz CPUs each. Two independent software implementations were used to ensure correctness of the graphs. One implementation was used to save the graph to disk, another implementation was used to generate and verify the complete degree distribution for each graph.

\section{Components and degree sequence}

Tables 1 and 2 provide a summary of the basic statistics for our switching graphs. Figure 1 shows the graphs (a) $\mathscr{G}_{8}^{\mathrm{i}}=\mathscr{G}_{8}^{\mathrm{f}}$ and (b) $\mathscr{G}_{8}^{\mathrm{v}}=\mathscr{G}_{8}^{\mathrm{fv}}$. The vertices are numbered in the order given in [1, Table 5.30] and [21]. For $n>8$ it is clearly impractical to draw our switching graphs.

Next, we discuss the components in our switching graphs, starting with the isolated vertices. In every case where isolated vertices arise, it is because there are no switchings available (recall the conventions outlined in Section 3; we do not allow switchings that produce a result that is isomorphic for a trivial reason). This contrasts to the "self- 


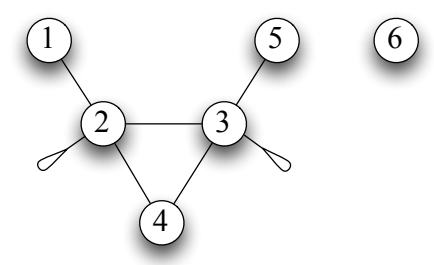

(a)

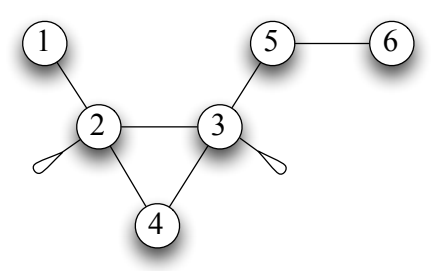

(b)

Figure 1: The switching graphs (a) $\mathscr{G}_{8}^{\mathrm{i}}=\mathscr{G}_{8}^{\mathrm{f}}$ and (b) $\mathscr{G}_{8}^{\mathrm{v}}=\mathscr{G}_{8}^{\mathrm{fv}}$

switching" isolated vertices encountered in [23], where switchings were available but none of them escaped the isomorphism class.

The graphs $\mathscr{G}_{8}^{\mathrm{v}}=\mathscr{G}_{8}^{\mathrm{fv}}$ and $\mathscr{G}_{10}^{\mathrm{v}}=\mathscr{G}_{10}^{\mathrm{fv}}$ do not have isolated vertices. In $\mathscr{G}_{12}^{\mathrm{fv}}$ there are two isolated vertices, and these are explained by Corollary 2. In $\mathscr{G}_{12}^{\mathrm{v}}$ there are eight isolated vertices, including the two that are isolated vertices in $\mathscr{G}_{12}^{\mathrm{fv}}$. Of the other six, the most symmetric is an example whose automorphism group is $\operatorname{PSL}(2,11)$. It is generated by the Mullin-Nemeth starter

$$
\{(1,2),(3,6),(4,8),(5,10),(9,7)\}
$$

over $\mathbb{Z}_{11}$. There is also an isolated vertex from the one-factorisation induced by the even starter

$$
\{(2,3),(4,8),(5,7),(6,9)\}
$$

over $\mathbb{Z}_{10}$. See $[1,21]$ for details on starters and the factorisations they induce. The only vertices of degree 1 in $\mathscr{G}_{12}^{\mathrm{fv}}$ are $(2),(3)$ and the $\mathrm{P} 1 \mathrm{~F}$ ' $\mathrm{D}$ ' from [18], which is also starterinduced. The $\mathrm{P} 1 \mathrm{~F}$ ' $\mathrm{D}$ ' is the only vertex of degree 1 in $\mathscr{G}_{12}^{\mathrm{v}}$.

The five isolated vertices in $\mathscr{G}_{12}^{\mathrm{f}}$ correspond to the P1Fs, which necessarily have no factor switchings available. Similarly, both $\mathscr{G}_{8}^{\mathrm{i}}=\mathscr{G}_{8}^{\mathrm{f}}$ and $\mathscr{G}_{10}^{\mathrm{i}}=\mathscr{G}_{10}^{\mathrm{f}}$ have a unique isolated vertex, and these are the P1Fs.

In $\mathscr{G}_{12}^{\mathrm{i}}$ there are 185 isolated vertices, 12 components of size 2 and two large components. All of the isolated vertices arise from one-factorisations that contain no 4-cycles, as characterised by Theorem 2. The 12 components of size 2 arise from Latin squares $U_{\mathscr{F}}$ which have precisely $68=\left(\begin{array}{l}n \\ 2\end{array}\right)+2$ intercalates, meaning that $\mathscr{F}$ has a single 4 -cycle switch available. Undergoing this switch produces no options for new switchings, other than to switch back to $\mathscr{F}$.

We use the informal term "giant component" to describe a component whose size is large relative to the whole graph. We say that a giant component is odd (respectively even) if the vertices in the giant component are all odd (respectively even). A priori, there is no reason why there could not be more than one giant component of a given parity but, like in [23], this did not happen in the cases we studied.

In $\mathscr{G}_{n}^{\mathrm{i}}$ and $\mathscr{G}_{n}^{\mathrm{f}}$ for $n \in\{8,10,12\}$ we know from Theorem 1 that there will be at least two components. In $\mathscr{G}_{8}^{\mathrm{i}}=\mathscr{G}_{8}^{\mathrm{f}}$ the isolated vertex from the $\mathrm{P} 1 \mathrm{~F}$ is the only vertex of odd parity. The even vertices in this case form a single component. In $\mathscr{G}_{10}^{\mathrm{i}}=\mathscr{G}_{10}^{\mathrm{f}}$ the isolated vertex from the P1F has odd parity. The other 200 odd vertices form a giant component, and the 195 even vertices form a separate giant component. 
In $\mathscr{G}_{12}^{\mathrm{f}}$ there are four even P1Fs and one odd P1F (the odd P1F is the one labelled ' $D$ ' in [18]) that form isolated vertices and the remaining vertices form two giant components. The odd giant component has order 263007611 and the even giant component has order 263908004. In $\mathscr{G}_{12}^{\mathrm{i}}$ there are 197 small components (as discussed above), plus two giant components. The odd giant component has order 263007524 and the even giant component has order 263907887.

All of the non-isolated vertices form a single giant component in both $\mathscr{G}_{n}^{\mathrm{v}}$ and $\mathscr{G}_{n}^{\mathrm{fv}}$ for each $n \in\{8,10,12\}$. Further properties of the giant components for all of our switching graphs will be discussed in Section 8 .

Next we specify the distribution of degrees in our switching graphs. In each case we list the degrees that are present, with a subscript indicating how many vertices possess the given degree. We always ignore loops when counting the degree of a vertex.

For $\mathscr{G}_{8}^{\mathrm{i}}=\mathscr{G}_{8}^{\mathrm{f}}$, as shown in Figure $1(\mathrm{a})$, the degrees are $\left[0_{1}, 1_{2}, 2_{1}, 3_{2}\right]$. Meanwhile, $\mathscr{G}_{8}^{\mathrm{v}}=\mathscr{G}_{8}^{\mathrm{fv}}$ is shown in Figure $1(\mathrm{~b})$. It is identical to $\mathscr{G}_{8}^{\mathrm{i}}=\mathscr{G}_{8}^{\mathrm{f}}$ except for the extra edge $(5,6)$. Hence the degrees in $\mathscr{G}_{8}^{\mathrm{v}}=\mathscr{G}_{8}^{\mathrm{fv}}$ are $\left[1_{2}, 2_{2}, 3_{2}\right]$.

For the larger graphs the most common degree will be shown in bold. For $\mathscr{G}_{10}^{\mathrm{i}}=\mathscr{G}_{10}^{\mathrm{f}}$ the degrees are

$$
\left[0_{1}, 1_{7}, 2_{9}, 3_{13}, 4_{14}, 5_{19}, 6_{37}, 7_{51}, 8_{38}, 9_{49}, 10_{48}, 11_{46}, 12_{37}, 13_{18}, 14_{5}, 15_{3}, 16_{1}\right],
$$

whereas for $\mathscr{G}_{10}^{\mathrm{v}}=\mathscr{G}_{10}^{\mathrm{fv}}$ they are

$$
\begin{aligned}
& {\left[2_{3}, 3_{1}, 4_{1}, 5_{3}, 6_{1}, 7_{4}, 8_{5}, 9_{4}, 10_{3}, 11_{7}, 12_{5}, 13_{5}, 14_{11}, 15_{5}, 16_{5}, 17_{9}, 18_{7}, 19_{7}, 20_{5}, 21_{6}, 22_{8}, 23_{9},\right.} \\
& 24_{11}, 25_{16}, 26_{18}, 27_{25}, 28_{26}, 29_{20}, \mathbf{3 0}_{\mathbf{2 8}}, 31_{22}, 32_{18}, 33_{12}, 34_{15}, 35_{25}, 36_{17}, 37_{11}, 38_{7}, 39_{4}, 40_{2}, \\
& \left.41_{3}, 42_{1}, 44_{1}\right] .
\end{aligned}
$$

The vertex of maximum degree in $\mathscr{G}_{10}^{\mathrm{i}}=\mathscr{G}_{10}^{\mathrm{f}}$ is represented by $A$ and the vertex of maximum degree in $\mathscr{G}_{10}^{\mathrm{v}}=\mathscr{G}_{10}^{\mathrm{fv}}$ is represented by $B$, where

$$
A=\left[\begin{array}{cccccccccc}
. & 1 & 2 & 3 & 4 & 5 & 6 & 9 & 7 & 8 \\
1 & . & 3 & 2 & 5 & 4 & 7 & 6 & 8 & 9 \\
2 & 3 & . & 1 & 6 & 8 & 9 & 4 & 5 & 7 \\
3 & 2 & 1 & . & 7 & 9 & 8 & 5 & 6 & 4 \\
4 & 5 & 6 & 7 & . & 1 & 2 & 8 & 9 & 3 \\
5 & 4 & 8 & 9 & 1 & . & 3 & 7 & 2 & 6 \\
6 & 7 & 9 & 8 & 2 & 3 & . & 1 & 4 & 5 \\
9 & 6 & 4 & 5 & 8 & 7 & 1 & . & 3 & 2 \\
7 & 8 & 5 & 6 & 9 & 2 & 4 & 3 & . & 1 \\
8 & 9 & 7 & 4 & 3 & 6 & 5 & 2 & 1 & \cdot
\end{array}\right], \quad B=\left[\begin{array}{cccccccccc}
\cdot & 1 & 2 & 4 & 3 & 7 & 9 & 6 & 5 & 8 \\
1 & . & 3 & 2 & 4 & 8 & 6 & 5 & 9 & 7 \\
2 & 3 & . & 1 & 5 & 9 & 8 & 7 & 6 & 4 \\
4 & 2 & 1 & . & 6 & 3 & 7 & 9 & 8 & 5 \\
3 & 4 & 5 & 6 & . & 1 & 2 & 8 & 7 & 9 \\
7 & 8 & 9 & 3 & 1 & . & 5 & 4 & 2 & 6 \\
9 & 6 & 8 & 7 & 2 & 5 & . & 1 & 4 & 3 \\
6 & 5 & 7 & 9 & 8 & 4 & 1 & . & 3 & 2 \\
5 & 9 & 6 & 8 & 7 & 2 & 4 & 3 & . & 1 \\
8 & 7 & 4 & 5 & 9 & 6 & 3 & 2 & 1 & \cdot
\end{array}\right] .
$$

Vertex $A$ has degree 16 and lies in the even giant component. The maximum degree in the odd giant component is 14 . It is achieved uniquely by the factorisation obtained from $A$ by switching within the two shaded row cycles and the corresponding column cycles. 
For $\mathscr{G}_{12}^{\mathrm{i}}, \mathscr{G}_{12}^{\mathrm{f}}, \mathscr{G}_{12}^{\mathrm{v}}$ and $\mathscr{G}_{12}^{\mathrm{fv}}$ the degree distributions are, respectively,

$\left[0_{185}, 1_{2349}, 2_{16304}, 3_{77663}, 4_{284620}, 5_{833810}, 6_{2042666}, 7_{4299667}, 8_{7942982}, 9_{13107750}, 1_{19571405}\right.$, $11_{26738716}, 12_{33769922}, 13_{39750900}, 14_{43930880}, \mathbf{1 5}_{\mathbf{4 5 8 5 8 9 8 9}}, 16_{45489647}, 17_{43069048}, 18_{39134962}$, $19_{34233441}, 20_{28952884}, 21_{23744049}, 22_{18946543}, 23_{14743824}, 24_{11228328}, 25_{8378168}, 26_{6141814}$, $27_{4432832}, 28_{3157063}, 29_{2220804}, 30_{1543457}, 31_{1065536}, 32_{729428}, 33_{492404}, 34_{332116}, 35_{221911}$, $36_{147703}, 37_{98028}, 38_{64108}, 39_{42464}, 40_{27329}, 41_{17678}, 42_{11328}, 43_{7438}, 44_{4545}, 45_{2975}, 46_{1859}$, $\left.47_{1254}, 48_{697}, 49_{456}, 50_{257}, 51_{173}, 52_{101}, 53_{56}, 54_{34}, 55_{24}, 56_{22}, 57_{8}, 58_{10}, 59_{4}, 61_{1}, 62_{1}\right]$,

$\left[0_{5}, 1_{7}, 2_{43}, 3_{157}, 4_{550}, 5_{2601}, 6_{10565}, 7_{37442}, 8_{117101}, 9_{321682}, 10_{776568}, 11_{1684050}, 12_{3301142}\right.$, $13_{5902616}, 14_{9666413}, 15_{14625338}, 16_{20552981}, 17_{26993491}, 18_{33265712}, 19_{38639504}, 20_{42478477}$, $\mathbf{2 1}_{\mathbf{4 4 3 4 7 7 0 4}}, 22_{44115238}, 23_{42018608}, 24_{38378969}, 25_{33769798}, 26_{28680623}, 27_{23612973}, 28_{18874636}$, $29_{14707259}, 30_{11189700}, 31_{8336019}, 32_{6097607}, 33_{4385597}, 34_{3113445}, 35_{2181644}, 36_{1514124}, 37_{1041153}$, $38_{709627}, 39_{482847}, 40_{324294}, 41_{218694}, 42_{146955}, 43_{97880}, 44_{64972}, 45_{43118}, 46_{28753}, 47_{19157}$, $48_{12591}, 49_{8432}, 50_{5672}, 51_{3669}, 52_{2526}, 53_{1651}, 54_{1066}, 55_{675}, 56_{532}, 57_{325}, 58_{198}, 59_{152}$, $\left.60_{86}, 61_{62}, 62_{55}, 63_{35}, 64_{11}, 65_{19}, 66_{9}, 67_{10}, 68_{3}, 71_{1}, 72_{1}\right]$,

$\left[0_{8}, 1_{1}, 2_{1}, 3_{4}, 4_{3}, 5_{3}, 6_{13}, 7_{9}, 8_{26}, 9_{24}, 10_{36}, 11_{55}, 12_{62}, 13_{75}, 14_{104}, 15_{95}, 16_{156}, 17_{151}, 18_{230}, 19_{287}\right.$, $20_{458}, 21_{600}, 22_{817}, 23_{1166}, 24_{1523}, 25_{2086}, 26_{2804}, 27_{3546}, 28_{4556}, 29_{5553}, 30_{6874}, 31_{8754}, 32_{11064}$, $33_{14000}, 34_{18913}, 35_{25536}, 36_{36126}, 37_{51802}, 38_{75332}, 39_{109509}, 40_{159050}, 41_{231538}, 42_{331250}, 43_{467314}$, $44_{649999}, 45_{893153}, 46_{1200769}, 47_{1595624}, 48_{2080982}, 49_{2675253}, 50_{3384643}, 51_{4217853}, 52_{5175069}$, $53_{6261511}, 54_{7464257}, 55_{8772795}, 56_{10166968}, 57_{11629681}, 58_{13119885}, 59_{14604149}, 60_{16057915}, 61_{17426729}$, $62_{18690775}, 63_{19785922}, 64_{20710419}, 65_{21416587}, 66_{21890813}, \mathbf{6 7}_{\mathbf{2 2 1 2 3 5 7 9}}, 68_{22102157}, 69_{21840281}$, $70_{21348774}, 71_{20645760}, 72_{19758702}, 73_{18720875}, 74_{17549429}, 75_{16284047}, 76_{14967415}, 77_{13625496}$, $78_{12291150}, 79_{10975437}, 80_{9720914}, 81_{8531212}, 82_{7414933}, 83_{6393414}, 84_{5471448}, 85_{4642686}, 86_{3906395}$, $87_{3264170}, 88_{2708743}, 89_{2228624}, 90_{1822311}, 91_{1481582}, 92_{1195512}, 93_{959323}, 94_{765916}, 95_{606635}$, $96_{478587}, 97_{376282}, 98_{293266}, 99_{228990}, 100_{176451}, 101_{136004}, 102_{104449}, 103_{80346}, 104_{60924}, 105_{46752}$, $106_{35274}, 107_{26678}, 108_{20186}, 109_{15248}, 110_{11314}, 111_{8529}, 112_{6513}, 113_{4849}, 114_{3769}, 115_{2760}$, $116_{2059}, 117_{1569}, 118_{1175}, 119_{885}, 120_{650}, 121_{453}, 122_{381}, 123_{285}, 124_{197}, 125_{151}, 126_{122}, 127_{88}$, $\left.128_{74}, 129_{42}, 130_{33}, 131_{31}, 132_{15}, 133_{14}, 134_{14}, 135_{9}, 136_{6}, 138_{5}, 139_{2}, 140_{1}, 142_{1}, 143_{1}, 148_{1}\right]$,

$\left[0_{2}, 1_{3}, 4_{2}, 5_{4}, 6_{1}, 7_{4}, 8_{13}, 9_{5}, 10_{18}, 11_{16}, 12_{27}, 13_{22}, 14_{38}, 15_{56}, 16_{52}, 17_{61}, 18_{70}, 19_{82}, 20_{110}, 21_{152}\right.$, $22_{162}, 23_{227}, 24_{263}, 25_{323}, 26_{355}, 27_{457}, 28_{630}, 29_{782}, 30_{1061}, 31_{1427}, 32_{1899}, 33_{2642}, 34_{3372}, 35_{4359}$, $36_{5500}, 37_{6948}, 38_{8795}, 39_{11265}, 40_{15088}, 41_{20708}, 42_{28842}, 43_{41409}, 44_{60892}, 45_{90457}, 46_{134353}$, $47_{198630}, 48_{290532}, 49_{420246}, 50_{597495}, 51_{831667}, 52_{1141678}, 53_{1536857}, 54_{2033783}, 55_{2641765}, 56_{3382332}$, $57_{4243344}, 58_{5247615}, 59_{6384880}, 60_{7644953}, 61_{9020899}, 62_{10470455}, 63_{11991897}, 64_{13534110}, 65_{15062975}$, $66_{16543651}, 67_{17942975}, 68_{19185520}, 69_{20271429}, 70_{21142174}, 71_{21787241}, 72_{22185889}, \mathbf{7 3}_{\mathbf{2 2 3 3 8 0 9 4}}$, 
$74_{22227841}, 75_{21874873}, 76_{21286812}, 77_{20503033}, 78_{19537507}, 79_{18425748}, 80_{17205159}, 81_{15907831}$, $82_{14568899}, 83_{13217774}, 84_{11891863}, 85_{10583565}, 8_{9346717}, 87_{8193302}, 88_{7113039}, 89_{6131165}$, $90_{5242128}, 91_{4448668}, 92_{3750240}, 93_{3137782}, 94_{2611001}, 95_{2157404}, 96_{1771133}, 97_{1447951}, 98_{1177264}$, $99_{951677}, 100_{767042}, 101_{614116}, 102_{490456}, 103_{389056}, 104_{308307}, 105_{243370}, 106_{192076}, 107_{150357}$, $108_{118386}, 109_{92420}, 110_{72288}, 111_{56553}, 112_{43659}, 113_{33680}, 114_{26373}, 115_{20429}, 116_{16003}, 117_{12216}$, $118_{9452}, 119_{7470}, 120_{5587}, 121_{4399}, 122_{3457}, 123_{2673}, 124_{2165}, 125_{1690}, 126_{1231}, 127_{992}, 128_{773}$, $129_{584}, 130_{434}, 131_{331}, 132_{272}, 133_{204}, 134_{159}, 135_{133}, 136_{112}, 137_{78}, 138_{54}, 139_{42}, 140_{35}$, $\left.141_{11}, 142_{21}, 143_{14}, 144_{12}, 145_{13}, 146_{4}, 147_{4}, 148_{5}, 149_{2}, 151_{2}, 152_{1}, 156_{1}\right]$.

The vertices of maximum degree in $\mathscr{G}_{12}^{\mathrm{i}}$ and $\mathscr{G}_{12}^{\mathrm{f}}$ coincide, and are represented by $C$ below. The vertices of maximum degree in $\mathscr{G}_{12}^{\mathrm{v}}$ and $\mathscr{G}_{12}^{\mathrm{fv}}$ coincide, and are represented by $D$ below.

$$
C=\left[\begin{array}{cccccccccccc}
\cdot & 1 & 2 & 3 & 4 & 5 & 7 & 8 & 9 & 10 & 6 & 11 \\
1 & \cdot & 3 & 2 & 5 & 4 & 9 & 10 & 7 & 8 & 11 & 6 \\
2 & 3 & \cdot & 1 & 6 & 8 & 10 & 4 & 11 & 5 & 7 & 9 \\
3 & 2 & 1 & . & 7 & 6 & 4 & 9 & 5 & 11 & 8 & 10 \\
4 & 5 & 6 & 7 & . & 1 & 2 & 11 & 3 & 9 & 10 & 8 \\
5 & 4 & 8 & 6 & 1 & . & 11 & 2 & 10 & 3 & 9 & 7 \\
7 & 9 & 10 & 4 & 2 & 11 & \cdot & 1 & 8 & 6 & 3 & 5 \\
8 & 10 & 4 & 9 & 11 & 2 & 1 & . & 6 & 7 & 5 & 3 \\
9 & 7 & 11 & 5 & 3 & 10 & 8 & 6 & \cdot & 1 & 2 & 4 \\
10 & 8 & 5 & 11 & 9 & 3 & 6 & 7 & 1 & \cdot & 4 & 2 \\
6 & 11 & 7 & 8 & 10 & 9 & 3 & 5 & 2 & 4 & \cdot & 1 \\
11 & 6 & 9 & 10 & 8 & 7 & 5 & 3 & 4 & 2 & 1 & \cdot
\end{array}\right] \quad D=\left[\begin{array}{cccccccccccc}
\cdot & 9 & 4 & 5 & 6 & 8 & 11 & 10 & 7 & 1 & 2 & 3 \\
9 & . & 5 & 6 & 4 & 10 & 7 & 8 & 11 & 2 & 3 & 1 \\
4 & 5 & \cdot & 11 & 8 & 6 & 9 & 7 & 10 & 3 & 1 & 2 \\
5 & 6 & 11 & \cdot & 9 & 4 & 1 & 2 & 3 & 8 & 10 & 7 \\
6 & 4 & 8 & 9 & . & 5 & 2 & 3 & 1 & 7 & 11 & 10 \\
8 & 10 & 6 & 4 & 5 & \cdot & 3 & 1 & 2 & 9 & 7 & 11 \\
11 & 7 & 9 & 1 & 2 & 3 & \cdot & 5 & 4 & 10 & 6 & 8 \\
10 & 8 & 7 & 2 & 3 & 1 & 5 & . & 9 & 11 & 4 & 6 \\
7 & 11 & 10 & 3 & 1 & 2 & 4 & 9 & . & 6 & 8 & 5 \\
1 & 2 & 3 & 8 & 7 & 9 & 10 & 11 & 6 & . & 5 & 4 \\
2 & 3 & 1 & 10 & 11 & 7 & 6 & 4 & 8 & 5 & . & 9 \\
3 & 1 & 2 & 7 & 10 & 11 & 8 & 6 & 5 & 4 & 9 & \cdot
\end{array}\right]
$$

The factorisation $C$ has 13 sub one-factorisations of order 4 . The factorisation $D$ has 6 sub one-factorisations of order 4 , all of which include the first vertex. It also has $3 \mathrm{sub}$ one-factorisations of $K_{3,3}$ including two induced by the colours $1,2,3$. The presence of all these substructures forces there to be lots of short cycles and hence plenty of different switching operations available. Indeed, $C$ has the largest number of 4-cycles of any onefactorisation of $K_{12}$ with a trivial automorphism group (although it is not unique in this respect).

Factorisation $C$ has even parity. The maximum degrees in the odd giant components of $\mathscr{G}_{12}^{\mathrm{i}}$ and $\mathscr{G}_{12}^{\mathrm{f}}$ are 57 and 68, respectively. Both are achieved by the factorisation obtained from $C$ by switching within the two shaded row cycles and also the corresponding column cycles. There are two other vertices of degree 57 in the odd giant component of $\mathscr{G}_{12}^{\mathrm{i}}$, but in every other component discussed in this section the maximum degree was achieved by a unique vertex.

We close the section by giving, in Table 3, the degrees of the two best known onefactorisations, namely $\mathrm{GK}_{n}$ and $\mathrm{GA}_{n}$, in each switching graph. For the construction and basic properties of $\mathrm{GK}_{n}$ and $\mathrm{GA}_{n}$, see $[1,21]$. In particular, it is known that $\mathrm{GK}_{8}, \mathrm{GA}_{10}$ 
and $\mathrm{GK}_{12}$ are perfect and hence will be isolated vertices in 4-cycle switching or factorswitching graphs, by Corollary 1. We also know from [3] and Corollary 1 that $\mathrm{GK}_{12}$ is an isolated vertex in all four types of switching graph. Note that $\mathrm{GK}_{10}$ and $\mathrm{GA}_{10}$ are both odd, while $\mathrm{GK}_{8}, \mathrm{GA}_{8}, \mathrm{GK}_{12}$ and $\mathrm{GA}_{12}$ are even.

\begin{tabular}{|c|cc|cc|cccc|}
\hline & $\mathscr{G}_{8}^{\mathrm{i}}=\mathscr{G}_{8}^{\mathrm{f}}$ & $\mathscr{G}_{8}^{\mathrm{v}}=\mathscr{G}_{8}^{\mathrm{fv}}$ & $\mathscr{G}_{10}^{\mathrm{i}}=\mathscr{G}_{10}^{\mathrm{f}}$ & $\mathscr{G}_{10}^{\mathrm{v}}=\mathscr{G}_{10}^{\mathrm{fv}}$ & $\mathscr{G}_{12}^{\mathrm{i}}$ & $\mathscr{G}_{12}^{\mathrm{f}}$ & $\mathscr{G}_{12}^{\mathrm{v}}$ & $\mathscr{G}_{12}^{\mathrm{fv}}$ \\
\hline $\mathrm{GK}_{n}$ & 0 & 1 & 1 & 2 & 0 & 0 & 0 & 0 \\
$\mathrm{GA}_{n}$ & 3 & 3 & 0 & 2 & 15 & 23 & 33 & 41 \\
\hline
\end{tabular}

Table 3: Degree of $\mathrm{GK}_{n}$ and $\mathrm{GA}_{n}$

\section{Cliques}

In this section we report on the cliques that are present in each of our switching graphs. In each graph we describe at least one maximum clique. A count of all the cliques in our switching graphs is given in Tables 4 and 5 .

\begin{tabular}{crrrr}
\hline Order & $\mathscr{G}_{8}^{\mathrm{i}}=\mathscr{G}_{8}^{\mathrm{f}}$ & $\mathscr{G}_{8}^{\mathrm{v}}=\mathscr{G}_{8}^{\mathrm{fv}}$ & $\mathscr{G}_{10}^{\mathrm{i}}=\mathscr{G}_{10}^{\mathrm{f}}$ & $\mathscr{G}_{10}^{\mathrm{v}}=\mathscr{G}_{10}^{\mathrm{fv}}$ \\
\hline 1 & 6 & 6 & 396 & 396 \\
2 & 5 & 6 & 1667 & 5212 \\
3 & 1 & 1 & 304 & 7407 \\
4 & & & 17 & 2708 \\
5 & & & & 318 \\
6 & & & & 16 \\
\hline
\end{tabular}

Table 4: Counts of cliques in switching graphs for $n=8$ and $n=10$

\begin{tabular}{crrrr}
\hline Order & \multicolumn{1}{c}{$\mathscr{G}_{12}^{\mathrm{i}}$} & \multicolumn{1}{c}{$\mathscr{G}_{12}^{\mathrm{f}}$} & \multicolumn{1}{c}{$\mathscr{G}_{12}^{\mathrm{v}}$} & \multicolumn{1}{c}{$\mathscr{G}_{12}^{\mathrm{fv}}$} \\
\hline 1 & 526915620 & 526915620 & 526915620 & 526915620 \\
2 & 4329000960 & 5869920634 & 17972340034 & 19513133875 \\
3 & 581836142 & 583647049 & 8516263134 & 10569839154 \\
4 & 145002498 & 145104034 & 2154240016 & 2779036888 \\
5 & 909 & 1336 & 481374 & 2843745 \\
6 & 23 & 60 & 50431 & 340632 \\
7 & 1 & 5 & 5536 & 30952 \\
8 & & & 663 & 3514 \\
9 & & & & 222 \\
10 & & & & 28 \\
11 & & & & \\
\hline
\end{tabular}

Table 5: Counts of cliques in switching graphs for $n=12$ 
It is apparent from Figure 1 that the clique number of each of the switching graphs for $n=8$ is 3 , and the unique 3 -clique contains the vertices 2,3,4 as numbered in [21] and [1, Table 5.30].

In $\mathscr{G}_{10}^{\mathrm{i}}=\mathscr{G}_{10}^{\mathrm{f}}$ the clique number is 4 . A representative of one 4-clique is given by $E$ below. The remaining three vertices of the clique can be found from $E$ by performing the shaded factor-switchings.

$$
E=\left[\begin{array}{llllllllll}
\cdot & 1 & 2 & 3 & 4 & 5 & 6 & 8 & 9 & 7 \\
1 & \cdot & 3 & 2 & 5 & 4 & 9 & 6 & 7 & 8 \\
2 & 3 & \cdot & 1 & 6 & 8 & 7 & 4 & 5 & 9 \\
3 & 2 & 1 & . & 7 & 9 & 8 & 5 & 6 & 4 \\
4 & 5 & 6 & 7 & . & 1 & 2 & 9 & 8 & 3 \\
5 & 4 & 8 & 9 & 1 & . & 3 & 7 & 2 & 6 \\
6 & 9 & 7 & 8 & 2 & 3 & \cdot & 1 & 4 & 5 \\
8 & 6 & 4 & 5 & 9 & 7 & 1 & . & 3 & 2 \\
9 & 7 & 5 & 6 & 8 & 2 & 4 & 3 & \cdot & 1 \\
7 & 8 & 9 & 4 & 3 & 6 & 5 & 2 & 1 & \cdot
\end{array}\right] \quad F=\left[\begin{array}{cccccccccc}
\cdot & 1 & 2 & 3 & 5 & 9 & 8 & 7 & 6 & 4 \\
1 & \cdot & 3 & 2 & 4 & 8 & 9 & 6 & 7 & 5 \\
2 & 3 & \cdot & 1 & 8 & 7 & 4 & 5 & 9 & 6 \\
3 & 2 & 1 & \cdot & 9 & 4 & 6 & 8 & 5 & 7 \\
5 & 4 & 8 & 9 & \cdot & 6 & 7 & 2 & 3 & 1 \\
9 & 8 & 7 & 4 & 6 & . & 5 & 1 & 2 & 3 \\
8 & 9 & 4 & 6 & 7 & 5 & \cdot & 3 & 1 & 2 \\
7 & 6 & 5 & 8 & 2 & 1 & 3 & . & 4 & 9 \\
6 & 7 & 9 & 5 & 3 & 2 & 1 & 4 & \cdot & 8 \\
4 & 5 & 6 & 7 & 1 & 3 & 2 & 9 & 8 & \cdot
\end{array}\right]
$$

In $\mathscr{G}_{10}^{\mathrm{v}}=\mathscr{G}_{10}^{\mathrm{fv}}$ the clique number is 6 . A representative of one 6 -clique is given by $F$ above. The remaining 5 vertices of the clique can be found from $F$ by performing the shaded vertex-switchings. In both $E$ and $F$, and henceforth, switchings are indicated by shading half the entries that change, with the other half being symmetrically placed about the main diagonal.

In $\mathscr{G}_{12}^{\mathrm{i}}$ there is a unique clique of order 7 and no larger cliques. One of the onefactorisations involved in the 7 -clique is this:

$$
\left[\begin{array}{cccccccccccc}
. & 1 & 2 & 3 & 4 & 5 & 8 & 9 & 7 & 6 & 10 & 11 \\
1 & . & 3 & 2 & 5 & 4 & 9 & 8 & 6 & 7 & 11 & 10 \\
2 & 3 & . & 1 & 6 & 7 & 10 & 11 & 9 & 8 & 5 & 4 \\
3 & 2 & 1 & . & 7 & 6 & 11 & 10 & 8 & 9 & 4 & 5 \\
4 & 5 & 6 & 7 & . & 1 & 2 & 3 & 10 & 11 & 9 & 8 \\
5 & 4 & 7 & 6 & 1 & . & 3 & 2 & 11 & 10 & 8 & 9 \\
8 & 9 & 10 & 11 & 2 & 3 & . & 1 & 5 & 4 & 7 & 6 \\
9 & 8 & 11 & 10 & 3 & 2 & 1 & . & 4 & 5 & 6 & 7 \\
7 & 6 & 9 & 8 & 10 & 11 & 5 & 4 & . & 2 & 1 & 3 \\
6 & 7 & 8 & 9 & 11 & 10 & 4 & 5 & 2 & . & 3 & 1 \\
10 & 11 & 5 & 4 & 9 & 8 & 7 & 6 & 1 & 3 & . & 2 \\
11 & 10 & 4 & 5 & 8 & 9 & 6 & 7 & 3 & 1 & 2 & \cdot
\end{array}\right]
$$

A representative of each of the other classes in the clique can be found by switching the shaded intercalates. Each of the factorisations in the clique has 7 sub one-factorisations of order 4 , including the disjoint ones induced by the colours 1,2,3.

In $\mathscr{G}_{12}^{\mathrm{f}}$ there are 5 cliques of order 7 and no larger cliques. One of these cliques was present in $\mathscr{G}_{12}^{\mathrm{i}}$, as just described. 
In $\mathscr{G}_{12}^{\mathrm{v}}$ there are 663 cliques of order 8 and no larger cliques. Here we analyse just one of the 8-cliques. One of its vertices is this:

$$
\left[\begin{array}{cccccccccccc}
. & 1 & 2 & 3 & 4 & 5 & 8 & 9 & 6 & 11 & 7 & 10 \\
1 & . & 3 & 2 & 5 & 4 & 9 & 8 & 11 & 6 & 10 & 7 \\
2 & 3 & . & 1 & 6 & 7 & 10 & 11 & 4 & 9 & 8 & 5 \\
3 & 2 & 1 & . & 7 & 6 & 11 & 10 & 8 & 5 & 4 & 9 \\
4 & 5 & 6 & 7 & . & 1 & 2 & 3 & 9 & 10 & 11 & 8 \\
5 & 4 & 7 & 6 & 1 & . & 3 & 2 & 10 & 8 & 9 & 11 \\
8 & 9 & 10 & 11 & 2 & 3 & . & 1 & 5 & 7 & 6 & 4 \\
9 & 8 & 11 & 10 & 3 & 2 & 1 & . & 7 & 4 & 5 & 6 \\
6 & 11 & 4 & 8 & 9 & 10 & 5 & 7 & . & 1 & 2 & 3 \\
11 & 6 & 9 & 5 & 10 & 8 & 7 & 4 & 1 & . & 3 & 2 \\
7 & 10 & 8 & 4 & 11 & 9 & 6 & 5 & 2 & 3 & . & 1 \\
10 & 7 & 5 & 9 & 8 & 11 & 4 & 6 & 3 & 2 & 1 & .
\end{array}\right]
$$

Consider the following 7 vertex-switchings, shown by the shaded areas in (4).

\begin{tabular}{|l|ccccccc|}
\hline Vertex-switching & $\mathrm{a}$ & $\mathrm{b}$ & $\mathrm{c}$ & $\mathrm{d}$ & $\mathrm{e}$ & $\mathrm{f}$ & $\mathrm{g}$ \\
Rows & 3,4 & 7,8 & 9,10 & 9,10 & 11,12 & 11,12 & 11,12 \\
Columns & 1,2 & 5,6 & 1,2 & $3,4,5,6,7,8$ & 1,2 & $3,4,5,6,7,8$ & 9,10 \\
\hline
\end{tabular}

(Each vertex-switching of course also changes the corresponding positions above the main diagonal, which have not been shown.) Starting from (4) and undertaking these seven switchings takes us to the other seven vertices in the clique. We next argue that any combination of two of the switchings is isomorphic to one of the other switchings. For example, doing switchings $e$ and $f$ is clearly isomorphic to just doing switching $g$ (via the isomorphism which interchanges the last two vertices). We encode this by saying $\mathrm{e} \oplus \mathrm{f}=\mathrm{g}$. Since the switchings are disjoint involutions, whenever we prove that $x \oplus y=z$ it will follow that $x \oplus z=x \oplus x \oplus y=y$ and $y \oplus z=y \oplus x \oplus y=x$. Indeed, any two of $\{x, y, z\}$ combine to give the third, so we have a partial triple system. Remarkably, our seven switchings actually form a Steiner triple system! We have already argued for the triple efg. The triple cdg is similar, as $c \oplus d=g$ by the isomorphism that interchanges vertices 9 and 10. Also, $\mathrm{a} \oplus \mathrm{b}=\mathrm{g}$ by the isomorphism which exchanges colours 2 and 3. We also have $\mathrm{a} \oplus \mathrm{c}=\mathrm{e}$ by the isomorphism that applies $(3,4)(5,6)(7,8)(9,10)(11,12)$ to permute the vertices and $(4,5)(8,9)$ to permute the colours. So that gives us the first four triples of the STS $\{$ efg, cdg, abg, ace, adf, bcf, bde $\}$. From these the other triples can be deduced since $\mathrm{a} \oplus \mathrm{d}=\mathrm{c} \oplus \mathrm{e} \oplus \mathrm{d}=\mathrm{e} \oplus \mathrm{g}=\mathrm{f}, \mathrm{b} \oplus \mathrm{c}=\mathrm{b} \oplus \mathrm{a} \oplus \mathrm{e}=\mathrm{g} \oplus \mathrm{e}=\mathrm{f}$, and $\mathrm{d} \oplus \mathrm{b}=\mathrm{d} \oplus \mathrm{a} \oplus \mathrm{g}=\mathrm{f} \oplus \mathrm{g}=\mathrm{e}$. 
There is a unique clique of order 11 in $\mathscr{G}_{12}^{\mathrm{fv}}$, and no larger cliques. The following one-factorisation is not a member of the clique:

$$
\left[\begin{array}{cccccccccccc}
. & 2 & 6 & 1 & 3 & 4 & 10 & 7 & 9 & 5 & 8 & 11 \\
2 & . & 3 & 4 & 6 & 1 & 5 & 10 & 8 & 7 & 11 & 9 \\
6 & 3 & . & 5 & 1 & 2 & 8 & 4 & 10 & 11 & 9 & 7 \\
1 & 4 & 5 & . & 2 & 3 & 7 & 9 & 11 & 10 & 6 & 8 \\
3 & 6 & 1 & 2 & . & 5 & 9 & 11 & 7 & 8 & 10 & 4 \\
4 & 1 & 2 & 3 & 5 & . & 11 & 8 & 6 & 9 & 7 & 10 \\
10 & 5 & 8 & 7 & 9 & 11 & . & 6 & 4 & 2 & 1 & 3 \\
7 & 10 & 4 & 9 & 11 & 8 & 6 & . & 5 & 3 & 2 & 1 \\
9 & 8 & 10 & 11 & 7 & 6 & 4 & 5 & . & 1 & 3 & 2 \\
5 & 7 & 11 & 10 & 8 & 9 & 2 & 3 & 1 & . & 4 & 6 \\
8 & 11 & 9 & 6 & 10 & 7 & 1 & 2 & 3 & 4 & . & 5 \\
11 & 9 & 7 & 8 & 4 & 10 & 3 & 1 & 2 & 6 & 5 & .
\end{array}\right]
$$

However, the vertices in the clique can be obtained by replacing the shaded subsquares by any one of the 11 other possibilities.

\section{Radius and diameter}

In a connected graph the eccentricity of a vertex $u$ is the maximum over all vertices $v$ of the distance from $u$ to $v$. The radius and diameter of a connected graph are respectively the minimum and maximum eccentricity of the vertices in the graph. In this section we study these parameters in each component of our switching graphs. If the radius of a component is $r$ it means that there is some vertex $u$ such that every other vertex in the component can be reached in $r$ or fewer switchings from $u$, and at least one vertex requires $r$ switchings. If the diameter is $d$ then it means that every pair of vertices can be joined by a sequence of $d$ or fewer switchings and there are two vertices that require this many. Both the radius and diameter provide information of practical relevance if it is desired to find a sequence of switchings to convert one one-factorisation into another.

Before giving our results, let us briefly discuss the computational aspects of studying the radius and the diameter of large graphs. For a given vertex $x$ in a given graph, we can compute the eccentricity of the vertex in its connected component using breadth-first search. Due to the size of our graphs, we find it convenient to use a variant of breadth-first search that maintains a bit map of the $N$ vertices, one bit for each vertex. We say that a vertex is marked if its bit is 1 ; otherwise the vertex is unmarked. Initially only the vertex $x$ is marked. We then run a sequence of linear passes $d=1,2, \ldots$ through the edge list of the graph, so that in each pass for each edge $\{y, z\}$ we check whether exactly one of the vertices is marked, and if so, we mark the unmarked vertex. The vertices that become marked on pass $d$ are exactly the vertices at distance $d$ from $x$ in the connected component that contains $x$. The algorithm terminates when no new vertices become marked during pass $d$, at which point we know that the eccentricity of $x$ in its connected component 
is $d-1$. To study the radius and diameter, our approach requires us to run multiple breadth-first searches from different sources $x$; in practice we accomplish this by running searches from 32 distinct $x$ in parallel so that the bit maps of the distinct $x$ are interleaved in memory, enabling efficient use of cache-memories during the passes through the edge list. With this optimization in place, we can run 32 breadth-first searches in parallel to recover the eccentricity and the balls of each radius around each vertex on a single node in about five core-hours; here it should be noted that due to the size of the graphs, each pass reads the edge list of the graph from disk and does not store it in main memory.

For $n<12$ it is simple to compute the eccentricity of every vertex in our switching graphs. To compute upper and lower bounds for the radius and diameter when $n=12$, we proceed as follows. First, the eccentricity of any given $x$ is (i) an upper bound for the radius of the component of $x$, and (ii) a lower bound for the diameter of the component of $x$. Thus, such upper and lower bounds may be recovered by carefully choosing a set of vertices $x$ and running breadth-first search. In practice we did this for a few thousand vertices that were notable for some reason, such as being close to extremal in terms of their degree, having a large automorphism group, or being in the largest clique. We also tested a selection of randomly chosen vertices and some vertices at maximum distance from vertices that we found had high eccentricity. This enabled us to form hypotheses about what we thought the radius and diameter of each component was.

We were then able to confirm our guesses for the radii in all cases, by the following approach. Denote by $B_{d}(x)$ the set of all vertices that are at distance at most $d$ from $x$. Suppose that we have a set of vertices $X=\left\{x_{1}, x_{2}, \ldots, x_{k}\right\}$, all in the same component, such that $B_{d}\left(x_{1}\right) \cap B_{d}\left(x_{2}\right) \cap \cdots \cap B_{d}\left(x_{k}\right)$ is empty. Since any vertex of eccentricity at most $d$ must be in $B_{d}(x)$ for all $x$, there is no such vertex. Hence, the radius of the component containing $X$ must be at least $d+1$. In particular, if this lower bound matches the upper bound established earlier, then we have recovered the radius of the component. The set of vertices $X$ was built in an ad-hoc way, starting with some vertices from our sample that had comparatively high eccentricity. We then iterated as follows:

1. Find $\cap_{x \in X} B_{d}(x)$. If this intersection is empty then we are done, so assume there are vertices in it. Select a few at random to form a set $Y$.

2. Do breadth first searches from the vertices in $Y$, recording the "antipodal" vertices, i.e. the ones that are at maximum distance from the initial vertex.

3. Augment $X$ by adding the vertices that appear most frequently among the lists of antipodal vertices from step 2 .

4. Return to step 1.

In each case, after perhaps half-a-dozen iterations of this loop, we obtained a set $X$ with $|X|<100$ such that $X$ provided witness to a lower bound for the radius that matched what we know is achieved. Unfortunately we have no analogous procedure for finding the diameter by executing a breadth-first searches from a manageable number of vertices. 


\begin{tabular}{clrr}
\hline Graph & Component & Radius & Diameter \\
\hline $\mathscr{G}_{10}^{\mathrm{i}}=\mathscr{G}_{10}^{\mathrm{f}}$ & even giant & 4 & 6 \\
& odd giant & 5 & 10 \\
$\mathscr{G}_{10}^{\mathrm{v}}=\mathscr{G}_{10}^{\mathrm{fv}}$ & giant & 3 & 6 \\
\hline $\mathscr{G}_{12}^{\mathrm{i}}$ & even giant & 15 & $21-30$ \\
& odd giant & 14 & $20-28$ \\
$\mathscr{G}_{12}^{\mathrm{f}}$ & even giant & 11 & $15-22$ \\
& odd giant & 11 & $13-22$ \\
$\mathscr{G}_{12}^{\mathrm{v}}$ & giant & 7 & $9-14$ \\
$\mathscr{G}_{12}^{\mathrm{fv}}$ & giant & 7 & $9-14$ \\
\hline
\end{tabular}

Table 6: Radius and diameter of the giant components

However, we suspect that the largest eccentricity that we encountered in our sample is close to the true diameter in each case.

Table 6 summarises our findings for the radius and diameter of the giant components in our switching graphs. For $n=12$ the table lists an interval for the diameter. The lower bound comes from an eccentricity that was computed in one of our breadth first searches, and the upper bound is twice the radius.

The eccentricity of each vertex in the switching graphs $\mathscr{G}_{8}^{\mathrm{i}}=\mathscr{G}_{8}^{\mathrm{f}}$ and $\mathscr{G}_{8}^{\mathrm{v}}=\mathscr{G}_{8}^{\mathrm{fv}}$ can be immediately determined from Figure 1.

The one-factorisation Example 3(a) achieves the maximum eccentricity in $\mathscr{G}_{10}^{\mathrm{v}}=\mathscr{G}_{10}^{\mathrm{fv}}$, which is 6. It also achieves the maximum eccentricity in the odd giant component of $\mathscr{G}_{10}^{\mathrm{i}}=\mathscr{G}_{10}^{\mathrm{f}}$, which is 10 . The only other vertex of eccentricity 10 is the P1F. The even giant component of $\mathscr{G}_{10}^{\mathrm{i}}=\mathscr{G}_{10}^{\mathrm{f}}$ has a much smaller diameter, namely 6 . A vertex of that eccentricity is the one obtained by the indicated vertex switching in Example 3(a). The minimum eccentricity in the even giant component of $\mathscr{G}_{10}^{\mathrm{i}}=\mathscr{G}_{10}^{\mathrm{f}}$ is 4 , which is achieved by the vertex of maximum degree (see Section 6). For the odd giant component the radius is 5, which is achieved by Example 3(b). Moreover, if the shaded factor-switching is undertaken, the resulting one-factorisation has the minimum eccentricity in $\mathscr{G}_{10}^{\mathrm{v}}=\mathscr{G}_{10}^{\mathrm{fv}}$, namely 3.

Finally, we consider the switching graphs for one-factorisations of $K_{12}$. The vertex of maximum degree in $\mathscr{G}_{12}^{\mathrm{v}}$ and $\mathscr{G}_{12}^{\mathrm{fv}}$, as given in Section 6, achieves the minimum eccentricity in the giant component, which is 7 in both graphs. The diameter of the giant component in both graphs is at least 9 . The pendant vertex corresponding to the starter induced $\mathrm{P} 1 \mathrm{~F}$ labelled ' $\mathrm{D}$ ' in [18] has eccentricity 9 in both cases.

In $\mathscr{G}_{12}^{\mathrm{f}}$ both giant components have radius 11. Vertices of this eccentricity (of both parities) may be found among the vertices that form the 11-clique in $\mathscr{G}_{12}^{\mathrm{fv}}$ as specified in (5). For the diameter of the giant components we have lower bounds of 15 for the even component (as achieved by (2)) and 13 for the odd component, as achieved by the factorisation induced by the starter $\{(1,3),(2,5),(4,9),(6,10),(7,8)\}$ in $\mathbb{Z}_{11}$. 
In $\mathscr{G}_{12}^{\mathrm{i}}$ the large even component has radius 15 and the large odd component has radius 14. Examples of vertices of these eccentricities are, respectively,

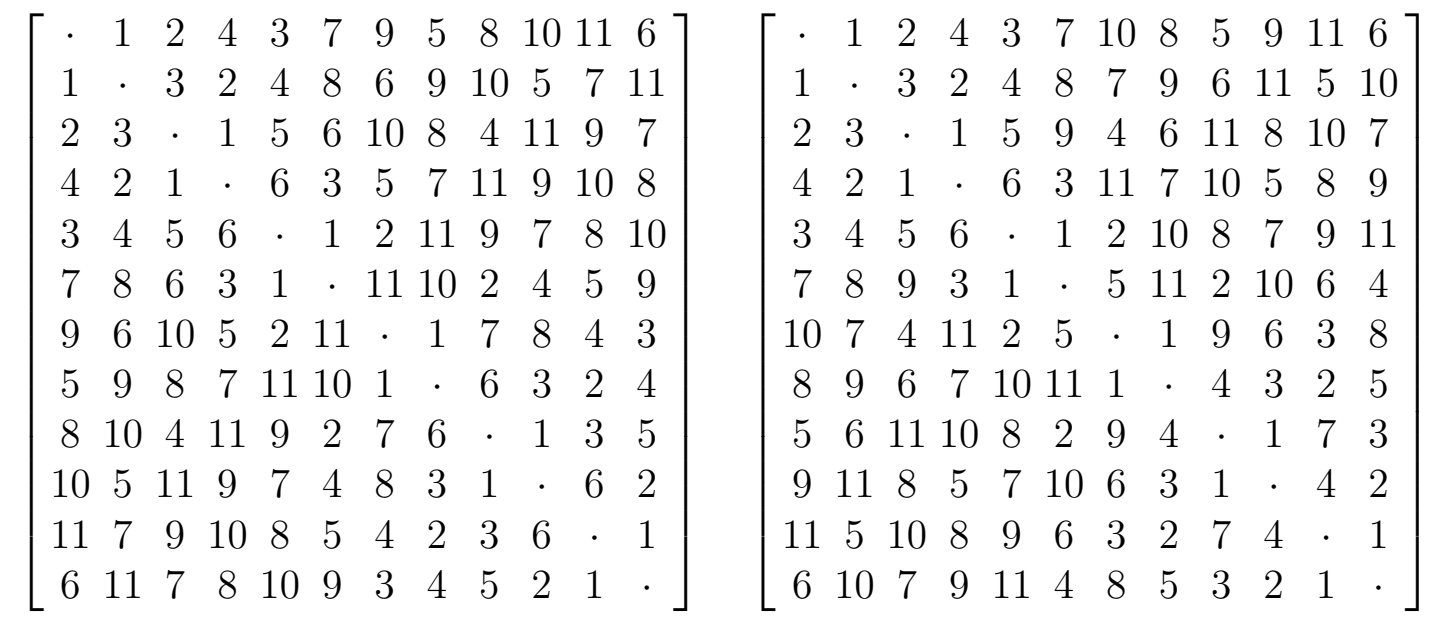

The diameters of the even and odd giant components in $\mathscr{G}_{12}^{\mathrm{i}}$ are at least 21 and 20 respectively. Examples achieving these eccentricities are:

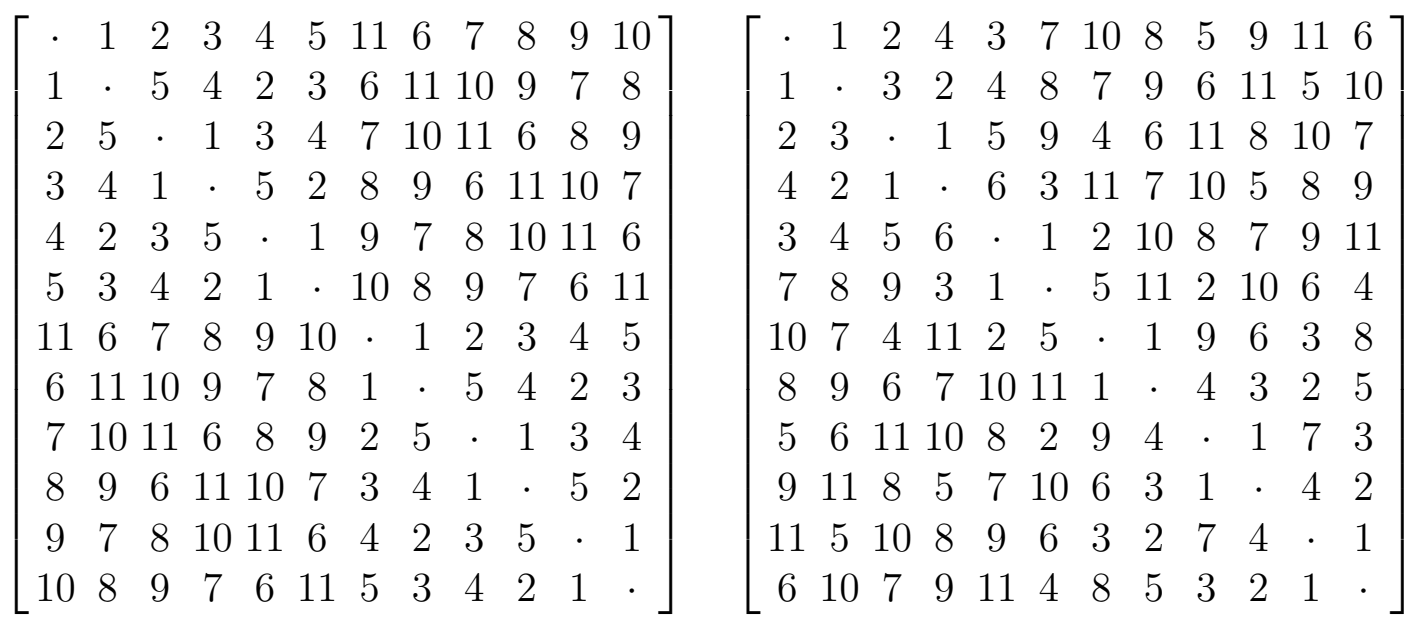

The left hand (even) example contains two disjoint sub one-factorisations of $K_{6}$, and has automorphism group of order 240. The right hand (odd) example is one of three vertices of degree 1 and eccentricity 20 in the odd giant component. All three have trivial automorphism groups.

We give the eccentricities of $\mathrm{GK}_{n}$ and $\mathrm{GA}_{n}$, in Table 7 .

\begin{tabular}{ccccccccc}
\hline & $\mathscr{G}_{8}^{\mathrm{i}}=\mathscr{G}_{8}^{\mathrm{f}}$ & $\mathscr{G}_{8}^{\mathrm{v}}=\mathscr{G}_{8}^{\mathrm{fv}}$ & $\mathscr{G}_{10}^{\mathrm{i}}=\mathscr{G}_{10}^{\mathrm{f}}$ & $\mathscr{G}_{10}^{\mathrm{v}}=\mathscr{G}_{10}^{\mathrm{fv}}$ & $\mathscr{G}_{12}^{\mathrm{i}}$ & $\mathscr{G}_{12}^{\mathrm{f}}$ & $\mathscr{G}_{12}^{\mathrm{v}}$ & $\mathscr{G}_{12}^{\mathrm{fv}}$ \\
\hline $\mathrm{GK}_{n}$ & 0 & 4 & 9 & 5 & 0 & 0 & 0 & 0 \\
$\mathrm{GA}_{n}$ & 2 & 3 & 0 & 6 & 18 & 13 & 8 & 9 \\
\hline
\end{tabular}

Table 7: Eccentricity of $\mathrm{GK}_{n}$ and $\mathrm{GA}_{n}$ 
We finish by stating a research challenge, which is to find local operations that are powerful enough to convert any one-factorisation of $K_{n}$ into any other one-factorisation of $K_{n}$. The switching operations discussed in this paper in general do not produce connected switching graphs. However, Pittenger [19] discovered "mappings" that can convert any Latin square into any other Latin square of the same order by changing only a small proportion of the structure at a time. It would be interesting to find similar operations for one-factorisations of $K_{n}$.

\section{References}

[1] L. D. Andersen, Factorizations of graphs, in: C. J. Colbourn and J. H. Dinitz (eds.), Handbook of Combinatorial Designs, 2nd edition, Chapman \& Hall/CRC, Boca Raton, 2007, pp. 740-755.

[2] M. Behbahani, C. Lam and P. R. J. Östergård, On triple systems and strongly regular graphs, J. Combin. Theory. Ser. A, 119 (2012), 1414-1426.

[3] D. Bryant, B. Maenhaut and I. M. Wanless, New families of atomic Latin squares and perfect one-factorisations, J. Combin. Theory Ser. A, 113 (2006), 608-624.

[4] N. J. Cavenagh, The theory and application of Latin bitrades: a survey, Math. Slovaca, 58 (2008), 691-718.

[5] C. J. Colbourn, A. D. Forbes, M. J. Grannell, T. S. Griggs, P. Kaski, P. R. J. Östergård, D. A. Pike and O. Pottonen, Properties of the Steiner triple systems of order 19, Electron. J. Combin., 17(1) (2010), \#R98.

[6] J.H. Dinitz, D. K. Garnick and B. D. McKay, There are 526,915,620 nonisomorphic one-factorizations of $K_{12}$, J. Combin. Des., 2 (1994), 273-285.

[7] V. Fack and B. D. McKay, A generalized switching method for combinatorial estimation, Australas. J. Combin., 39 (2007), 141-154.

[8] M. J. Grannell, T.S. Griggs and J.P. Murphy, Switching cycles in Steiner triple systems, Util. Math., 56 (1999), 3-21.

[9] M. T. Jacobson and P. Matthews, Generating uniformly distributed random Latin squares, J. Combin. Des., 4 (1996), 405-437.

[10] P. Kaski, V. Mäkinen and P. R. J. Östergård, The cycle switching graph of the Steiner triple systems of order 19 is connected, Graphs Combin., 27 (2011), 539-546.

[11] P. Kaski and P. R. J. Östergård, There are 1,132,835,421,602,062,347 nonisomorphic one-factorizations of $K_{14}$, J. Combin. Des., 17 (2009), 147-159.

[12] C. C. Lindner, E. Mendelsohn and A. Rosa, On the number of 1-factorizations of the complete graph, J. Combin. Theory Ser. B, 20 (1976), 265-282.

[13] B. M. Maenhaut and I. M. Wanless, Atomic Latin squares of order eleven, J. Combin. Des., 12 (2004), 12-34.

[14] B. D. McKay, Practical graph isomorphism, Congr. Numer., 30 (1981), 45-87. 
[15] B. D. McKay and A. Piperno, Practical Graph Isomorphism II, J. Symbolic Comput., 60 (2014), 94-112.

[16] M. Meszka, $k$-cycle free one-factorizations of complete graphs, Electron. J. Combin., 16(1) (2009), \#R3.

[17] P. R. J. Östergård, Switching codes and designs, Discrete Math., 312 (2012), 621-632.

[18] A. P. Petrenyuk and A. Y. Petrenyuk, Intersection of perfect 1-factorizations of complete graphs, Cybernetics, 16 (1980), 6-8.

[19] A. O. Pittenger, Mappings of Latin squares, Linear Algebra Appl., 261 (1997), 251268.

[20] D. S. Stones and I. M. Wanless, How not to prove the Alon-Tarsi conjecture, Nagoya Math. J., 205 (2012), 1-24.

[21] W. D. Wallis, One-factorizations, Kluwer Academic, Dordrecht, Netherlands, 1997.

[22] I. M. Wanless, Perfect factorisations of bipartite graphs and Latin squares without proper subrectangles, Electron. J. Combin., 6 (1999), \#R9.

[23] I. M. Wanless, Cycle switching in Latin squares, Graphs Combin., 20 (2004), 545-570.

[24] I. M. Wanless, Atomic Latin squares based on cyclotomic orthomorphisms, Electron. J. Combin., 12 (2005), \#R22.

[25] I. M. Wanless and E. C. Ihrig, Symmetries that Latin squares inherit from 1factorizations, J. Combin. Des., 13 (2005), 157-172. 